\title{
$H$-free graphs of large minimum degree
}

\author{
Noga Alon * Benny Sudakov ${ }^{\dagger}$
}

Submitted: Aug 16, 2005; Accepted: Feb 13, 2006; Published: Mar 7, 2006

Mathematics Subject Classification: 05C35

\begin{abstract}
We prove the following extension of an old result of Andrásfai, Erdős and Sós. For every fixed graph $H$ with chromatic number $r+1 \geq 3$, and for every fixed $\epsilon>0$, there are $n_{0}=n_{0}(H, \epsilon)$ and $\rho=\rho(H)>0$, such that the following holds. Let $G$ be an $H$-free graph on $n>n_{0}$ vertices with minimum degree at least $\left(1-\frac{1}{r-1 / 3}+\epsilon\right) n$. Then one can delete at most $n^{2-\rho}$ edges to make $G r$-colorable.
\end{abstract}

\section{Introduction}

Turán's classical Theorem [11] determines the maximum number of edges in a $K_{r+1}$-free graph on $n$ vertices. It easily implies that for $r \geq 2$, if a $K_{r+1}$-free graph on $n$ vertices has minimum degree at least $\left(1-\frac{1}{r}\right) n$, then it is $r$-colorable (in fact, it is a complete $r$-partite graph with equal color classes). The following stronger result has been proved by Andrásfai, Erdős and Sós [2].

Theorem 1.1 ([2]) If $G$ is a $K_{r+1}$-free graph of order $n$ with minimum degree $\delta(G)>$ $\left(1-\frac{1}{r-1 / 3}\right) n$ then $G$ is $r$-colorable.

The following construction shows that this is tight. Let $G$ be a graph whose vertex set is the disjoint union of $r+3$ sets $U_{1}, U_{2}, \ldots, U_{5}$ and $V_{1}, V_{2} \ldots, V_{r-2}$, in which $\left|U_{i}\right|=\frac{1}{3 r-1} n$ for all $i$ and $\left|V_{j}\right|=\frac{3}{3 r-1} n$ for all $j$. Each vertex of $V_{j}$ is adjacent to all vertices but the other members of $V_{j}$ and each vertex of $U_{i}$ is adjacent to all vertices of $U_{(i+1) \bmod 5}, U_{(i-1) \bmod 5}$

*Schools of Mathematics and Computer Science, Raymond and Beverly Sackler Faculty of Exact Sciences, Tel Aviv University, Tel Aviv 69978, Israel and IAS, Princeton, NJ 08540, USA. Email: nogaa@tau.ac.il. Research supported in part by a USA-Israeli BSF grant, by NSF grant CCR-0324906, by a Wolfensohn fund and by the State of New Jersey.

${ }^{\dagger}$ Department of Mathematics, Princeton University, Princeton, NJ 08544, USA. E-mail: bsudakov@math.princeton.edu. Research supported in part by NSF CAREER award DMS-0546523, NSF grant DMS-0355497, USA-Israeli BSF grant, and by an Alfred P. Sloan fellowship. 
and $\cup_{j} V_{j}$. All vertices in this graph have degree $\frac{3 r-4}{3 r-1} n=\left(1-\frac{1}{r-1 / 3}\right) n$ and it is easy to see that $G$ contains no $K_{r+1}$, and is not $r$-colorable.

Turán's result has been extended by Erdős-Stone [6] and by Erdős-Simonovits [4] showing that for $r \geq 2$, for any fixed graph $H$ of chromatic number $\chi(H)=r+1$ and for any fixed $\epsilon>0$, any $H$-free graph on $n$ vertices cannot have more than $\left(1-\frac{1}{r}+\epsilon\right)\left(\begin{array}{l}n \\ 2\end{array}\right)$ edges provided $n$ is sufficiently large as a function of $H$ and $\epsilon$. Moreover, it is known that if an $H$-free graph on a large number $n$ of vertices has at least $\left(1-\frac{1}{r}\right)\left(\begin{array}{l}n \\ 2\end{array}\right)$ edges, then one can delete $o\left(n^{2}\right)$ of its edges to make it $r$-colorable.

It therefore seems natural to try to extend Theorem 1.1 from complete graphs $K_{r+1}$ to general graphs $H$. Such an extension for critical graphs, i.e., $H$ which have an edge whose removal decreases its chromatic number, has been proved in [5]. In the present short paper we handle the general case. Our main results are the following. Let $K_{r+1}(t)$ be the complete $(r+1)$-partite graph with $t$ vertices in each vertex class.

Theorem 1.2 Let $r \geq 2, t \geq 1$ be integers and let $\epsilon>0$. Then there exist $n_{0}=$ $n_{0}(r, t, \epsilon)$ such that if $G$ is a $K_{r+1}(t)$-free graph of order $n \geq n_{0}$ with minimum degree $\delta(G) \geq\left(1-\frac{1}{r-1 / 3}+\epsilon\right) n$, then one can delete at most $O\left(n^{2-1 /\left(4 r^{2 / 3} t\right)}\right)$ edges to make $G$ $r$-colorable.

Corollary 1.3 Let $H$ be a fixed graph on $h$ vertices with chromatic number $r+1 \geq 3$, suppose $\epsilon>0$ and let $G$ be an $H$-free graph of sufficiently large order $n>n_{0}(h, \epsilon)$ with minimum degree $\delta(G) \geq\left(1-\frac{1}{r-1 / 3}+\epsilon\right) n$. Then one can delete at most $O\left(n^{2-1 /\left(4 r^{2 / 3} h\right)}\right)$ edges to make $G$ r-colorable.

As shown by the example above, the fraction $1-\frac{1}{r-1 / 3}=\frac{3 r-4}{3 r-1}$ is tight in general. It is also not difficult to see that indeed in general some $O\left(n^{2-\rho}\right)$ edges have to be deleted to make the graph $G r$-colorable, though the best possible value of $\rho=\rho\left(K_{r+1}(t)\right)$ may well be slightly better than the one we obtain. The problem of determining the behavior of the best possible value of $\rho$, as well as that of deciding if the $\epsilon n$-term can be replaced by $O(1)$, remain open.

A weaker version of Corollary 1.3 is proved in [1], where it is applied to prove the NP-hardness of various edge-deletion problems. This version asserts that there are some $\gamma=\gamma(H)>0$ and $\mu=\mu(H)>0$ so that the following holds. For any $H$-free graph $G$ on $n$ vertices with minimum degree at least $(1-\gamma) n$, one can delete $O\left(n^{2-\mu}\right)$ edges from $G$ to make it $r$-colorable. Theorem 1.2 supplies the asymptotically best possible value of $\gamma\left(K_{r+1}(t)\right)$ for all admissible $r$ and $t$.

\section{Proofs}

In this section we prove our main theorem. Let $G$ be a $K_{r+1}(t)$-free graph of order $n$ with minimum degree $\delta(G) \geq\left(1-\frac{1}{r-1 / 3}+\epsilon\right) n$. We assume throughout the proof that $n$ is sufficiently large. We first establish the following weaker bound. 
Lemma 2.1 $G$ can be made r-partite by deleting o $\left(n^{2}\right)$ edges.

The proof of this statement is a standard application of Szemerédi's Regularity Lemma and we refer the interested reader to the comprehensive survey of Komlós and Simonovits [8], which discusses various results proved by this powerful tool.

We start with a few definitions, most of which follow [8]. Let $G=(V, E)$ be a graph, and let $A$ and $B$ be two disjoint subsets of $V(G)$. If $A$ and $B$ are non-empty, define the density of edges between $A$ and $B$ by $d(A, B)=\frac{e(A, B)}{|A||B|}$. For $\gamma>0$ the pair $(A, B)$ is called $\gamma$-regular if for every $X \subset A$ and $Y \subset B$ satisfying $|X|>\gamma|A|$ and $|Y|>\gamma|B|$ we have $|d(X, Y)-d(A, B)|<\gamma$. An equitable partition of a set $V$ is a partition of $V$ into pairwise disjoint classes $V_{1}, \cdots, V_{k}$ of almost equal size, i.e., ||$V_{i}|-| V_{j}|| \leq 1$ for all $i, j$. An equitable partition of the set of vertices $V$ of $G$ into the classes $V_{1}, \cdots, V_{k}$ is called $\gamma$-regular if $\left|V_{i}\right| \leq \gamma|V|$ for every $i$ and all but at most $\gamma k^{2}$ of the pairs $\left(V_{i}, V_{j}\right)$ are $\gamma$-regular. The above partition is called totally $\gamma$-regular if all the pairs $\left(V_{i}, V_{j}\right)$ are $\gamma$-regular. The following celebrated lemma was proved by Szemerédi in [10].

Lemma 2.2 For every $\gamma>0$ there is an integer $M(\gamma)$ such that every graph of order $n>M(\gamma)$ has a $\gamma$-regular partition into $k$ classes, where $k \leq M(\gamma)$.

In order to apply the Regularity Lemma we need to show the existence of a complete multipartite subgraph in graphs with a totally $\gamma$-regular partition. This is established in the following well-known lemma, see, e.g., [8].

Lemma 2.3 For every $\eta>0$ and integers $r$, there exist $0<\gamma=\gamma(\eta, r, t)$ and $n_{0}=$ $n_{0}(\eta, r, t)$ with the following property. If $G$ is a graph of order $n>n_{0}$ and $\left(V_{1}, \cdots, V_{r+1}\right)$ is a totally $\gamma$-regular partition of vertices of $G$ such that $d\left(V_{i}, V_{j}\right) \geq \eta$ for all $i<j$, then $G$ contains a complete $(r+1)$-partite subgraph $K_{r+1}(t)$ with parts of size $t$.

Proof of Lemma 2.1. We use the Regularity Lemma given in Lemma 2.2. For every constant $0<\eta<\epsilon / 4$ let $\gamma=\gamma(\eta, r, t)<\eta^{2}$ be sufficiently small to guarantee that the assertion of Lemma 2.3 holds. Consider a $\gamma$-regular partition $\left(U_{1}, U_{2}, \ldots U_{k}\right)$ of $G$. Let $G^{\prime}$ be a new graph on the vertices $1 \leq i \leq k$ in which $(i, j)$ is an edge iff $\left(U_{i}, U_{j}\right)$ is a $\gamma$-regular pair with density at least $\eta$. Since $G$ is a $K_{r+1}(t)$-free graph, by Lemma $2.3, G^{\prime}$ contains no clique of size $r+1$. Call a vertex of $G^{\prime}$ good if there are at most $\eta k$ other vertices $j$ such that the pair $\left(U_{i}, U_{j}\right)$ is not $\gamma$-regular, otherwise call it bad. Since the number of non-regular pairs is at most $\gamma\left(\begin{array}{l}k \\ 2\end{array}\right) \leq \eta^{2} k^{2} / 2$ we have that all but at most $\eta k$ vertices are good. By the definition of "good" and by the assumption on the minimum degree of $G$, the degree of each good vertex in $G^{\prime}$ is at least $\left(1-\frac{1}{r-1 / 3}+\epsilon\right) k-2 \eta k-1$, since deletion of the edges from non-regular pairs and sparse pairs can decrease the degree by at most $\eta k$ each and the deletion of edges inside the sets $U_{i}$ can decrease it by 1 . By deleting all bad vertices we obtain a $K_{r+1}$-free graph on at most $k$ vertices with minimum degree at least

$$
\left(1-\frac{1}{r-1 / 3}+\epsilon\right) k-3 \eta k-1 \geq\left(1-\frac{1}{r-1 / 3}+\epsilon\right) k-4 \eta k>\left(1-\frac{1}{r-1 / 3}\right) k .
$$


Therefore, by the result of Andrásfai, Erdős and Sós [2] mentioned as Theorem 1.1 in the introduction, this graph is $r$-partite. This implies that to make $G r$-partite it suffices to delete at most $\gamma n^{2}+\eta n^{2}+(\eta n) \cdot n+k \cdot(n / k)^{2} \leq 3 \eta n^{2}+n^{2} / k=o\left(n^{2}\right)$ edges.

Consider a partition $\left(V_{1}, \ldots, V_{r}\right)$ of the vertices of $G$ into $r$ parts which maximizes the number of crossing edges between the parts. Then for every $x \in V_{i}$ and $j \neq i$ the number of neighbors of $x$ in $V_{i}$ is at most the number of its neighbors in $V_{j}$, as otherwise by shifting $x$ to $V_{j}$ we increase the number of crossing edges. By the above discussion, we have that this partition satisfies that $\sum_{i} e\left(V_{i}\right)=o\left(n^{2}\right)$. Call a vertex $x$ of $G$ typical if $x \in V_{i}$ has at most $\epsilon n / 2$ neighbors in $V_{i}$. Note that there are at most $o(n)$ non-typical vertices in $G$ and, in particular, every part $V_{i}$ contains a typical vertex. By definition, the degree of this vertex outside $V_{i}$ is at least $\left(\frac{3 r-4}{3 r-1}+\epsilon\right) n-\epsilon n / 2=\left(\frac{3 r-4}{3 r-1}+\epsilon / 2\right) n$ and at most $n-\left|V_{i}\right|$. Therefore, for all $1 \leq i \leq r$

$$
\begin{aligned}
& \left|V_{i}\right| \leq n-\left(\frac{3 r-4}{3 r-1}+\epsilon / 2\right) n=\left(\frac{3}{3 r-1}-\epsilon / 2\right) n \\
& \left|V_{i}\right| \geq n-\sum_{j \neq i}\left|V_{j}\right| \geq n-(r-1)\left(\frac{3}{3 r-1}-\epsilon / 2\right) n \geq\left(\frac{2}{3 r-1}+\epsilon / 2\right) n .
\end{aligned}
$$

Our next lemma reduces further the possible number of non-typical vertices in $G$.

Lemma 2.4 Each $V_{i}$ contains at most $O(1)$ non-typical vertices.

To prove this statement we need the following two claims.

Claim 2.5 Let $y_{1}, \ldots, y_{k}$ be an arbitrary set of $k \leq r-1$ typical vertices outside $V_{j}$, such that each $y_{i}$ belongs to a different part of the partition. Then $V_{j}$ contains at least $\frac{2}{3 r-1} n$ vertices adjacent to all vertices $y_{i}$.

Proof. It is enough to prove this statement for $k=r-1$, since the addition of $r-1-k$ typical vertices $y_{i}$ from the remaining parts can only decrease the size of the common neighborhood. Thus, without loss of generality, we assume that $V_{j}=V_{r}$ and $y_{i} \in V_{i}, 1 \leq$ $i \leq r-1$. Since every $y_{i}$ is a typical vertex it has at most $\epsilon n / 2$ neighbors in $V_{i}$ and hence at most $\epsilon n / 2+\left(n-\left|V_{i}\right|-\left|V_{r}\right|\right)$ neighbors outside $V_{r}$. This implies that the number of neighbors of $y_{i}$ in $V_{r}$ is at least

$$
\begin{aligned}
d_{V_{r}}\left(y_{i}\right) & \geq d\left(y_{i}\right)-\left((1+\epsilon / 2) n-\left|V_{i}\right|-\left|V_{r}\right|\right) \\
& \geq\left(\frac{3 r-4}{3 r-1}+\epsilon\right) n-\left((1+\epsilon / 2) n-\left|V_{i}\right|-\left|V_{r}\right|\right) \\
& >\left|V_{r}\right|+\left|V_{i}\right|-\frac{3}{3 r-1} n
\end{aligned}
$$

By definition, there are at most $\left|V_{r}\right|-d_{V_{r}}\left(y_{i}\right)<\frac{3}{3 r-1} n-\left|V_{i}\right|$ non-neighbors of $y_{i}$ in $V_{r}$. Delete from $V_{r}$ any vertex, which is not a neighbor of either $y_{1}, y_{2}, \ldots, y_{r-1}$. The 
remaining set is adjacent to every vertex $y_{i}$ and has size at least

$$
\begin{aligned}
\left|V_{r}\right|-\sum_{i}\left(\left|V_{r}\right|-d_{V_{r}}\left(y_{i}\right)\right) & >\left|V_{r}\right|-\sum_{i \leq r-1}\left(\frac{3}{3 r-1} n-\left|V_{i}\right|\right) \\
& =\sum_{i=1}^{r}\left|V_{i}\right|-(r-1) \frac{3}{3 r-1} n \\
& =n-\frac{3 r-3}{3 r-1} n=\frac{2}{3 r-1} n .
\end{aligned}
$$

Claim 2.6 For every non-typical vertex $x \in V_{i}$ there are at least $(\epsilon n / 3)^{r}$ cliques $y_{1}, \ldots, y_{r}$ of size $r$ such that $y_{j} \in V_{j}$ for all $1 \leq j \leq r$ and all vertices $y_{j}$ are adjacent to $x$.

Proof. Without loss of generality let $i=1$ and let $x \in V_{1}$ be a non-typical vertex. Since for every $j \neq 1$ the number of neighbors of $x$ in $V_{j}$ is at least as large as the number of its neighbors in $V_{1}$ we have that

$$
\begin{aligned}
d_{V_{j}}(x) & \geq \frac{d_{V_{j}}(x)+d_{V_{1}}(x)}{2} \geq \frac{1}{2}\left(\left(\frac{3 r-4}{3 r-1}+\epsilon\right) n-(r-2) \max _{i}\left|V_{i}\right|\right) \\
& >\frac{1}{2}\left(\left(\frac{3 r-4}{3 r-1}+\epsilon\right) n-(r-2) \frac{3}{3 r-1} n\right) \\
& =\left(\frac{1}{3 r-1}+\epsilon / 2\right) n .
\end{aligned}
$$

To construct the $r$-cliques satisfying the assertion of the claim, first observe, that since $x$ is non-typical it has at least $\epsilon n / 2$ neighbors in $V_{1}$ and at least $\epsilon n / 2-o(n)>\epsilon n / 3$ of these neighbors are typical. Choose $y_{1}$ to be an arbitrary typical neighbor of $x$ in $V_{1}$ and continue. Suppose at step $1 \leq k \leq r-1$ we already have a $k$-clique $y_{1}, \ldots, y_{k}$ such that $y_{i} \in V_{i}$ for all $i$ and all vertices $y_{i}$ are adjacent to $x$. Let $U_{k+1}$ be the set of common neighbors of $y_{1}, \ldots, y_{k}$ in $V_{k+1}$. Then, by the previous claim we have that $\left|U_{k+1}\right| \geq \frac{2}{3 r-1} n$. Therefore, there are at least

$$
d_{V_{k+1}}(x)+\left|U_{k+1}\right|-\left|V_{k+1}\right| \geq\left(\frac{1}{3 r-1}+\epsilon / 2\right) n+\frac{2}{3 r-1} n-\frac{3}{3 r-1} n=\epsilon n / 2
$$

common neighbors of the vertices $y_{i}$ and $x$ in $V_{k+1}$. Moreover, at least $\epsilon n / 2-o(n)>\epsilon n / 3$ of them are typical and we can choose $y_{k+1}$ to be any of them. Therefore at the end of the process we indeed obtained at least $(\epsilon n / 3)^{r} r$-cliques with the desired property.

Proof of Lemma 2.4. Suppose that the number of non-typical vertices in $V_{i}$ is at least $t(3 / \epsilon)^{r}$. Consider an auxiliary bipartite graph $F$ with parts $W_{1}, W_{2}$, where $W_{1}$ is the set of some $s=t(3 / \epsilon)^{r}$ non-typical vertices in $V_{i}, W_{2}$ is the family of all $n^{r} r$-element subsets of $V(G)$ such that $x \in W_{1}$ is adjacent to the subset $Y$ from $W_{2}$ iff $Y$ is an $r$-clique in 
$G$ with exactly one vertex in every $V_{j}$ and all vertices of $Y$ are adjacent to $x$. By the previous claim, $F$ has at least $e(F) \geq s(\epsilon n / 3)^{r}=t n^{r}$ edges and therefore the average degree of a vertex in $W_{2}$ is at least $d_{a v}=e(F) /\left|W_{2}\right|=e(F) / n^{r} \geq t$. By the convexity of the function $f(z)=\left(\begin{array}{c}z \\ t\end{array}\right)$, we can find $t$ vertices $x_{1}, \ldots, x_{t}$ in $W_{1}$ such that the number of their common neighbors in $W_{2}$ is at least

$$
m \geq \frac{\sum_{Y \in W_{2}}\left(\begin{array}{c}
d(Y) \\
t
\end{array}\right)}{\left(\begin{array}{l}
s \\
t
\end{array}\right)} \geq n^{r} \frac{\left(\begin{array}{c}
d_{a v} \\
t
\end{array}\right)}{s^{t}}=\Omega\left(n^{r}\right) .
$$

Thus we proved that $G$ contains $t$ vertices $X=\left\{x_{1}, \ldots, x_{t}\right\}$ and a family of $r$-cliques $\mathcal{C}$ of size $m=\Omega\left(n^{r}\right)$ such that every clique in $\mathcal{C}$ is adjacent to all vertices in $X$. Next we need the following well-known lemma which appears first implicitly in Erdős [3] (see also, e.g., [7]). It states that if an $r$-uniform hypergraph on $n$ vertices has $m=\Omega\left(n^{r}\right)$ edges, then it contains a complete $r$-partite $r$-uniform hypergraph with parts of size $t$. By applying this statement to $\mathcal{C}$, we conclude that there are $r$ disjoint set of vertices $A_{1}, \ldots, A_{r}$ each of size $t$ such that every $r$-tuple $a_{1}, \ldots, a_{r}$ with $a_{i} \in A_{i}$ forms a clique which is adjacent to all vertices in $X$. The restriction of $G$ to $X, A_{1}, \ldots, A_{r}$ forms a complete $(r+1)$-partite graph with parts of size $t$ each. This contradiction shows that there are less than $t(3 / \epsilon)^{r}=O(1)$ non-typical vertices in $V_{i}$ and completes the proof of the lemma.

Lemma 2.7 Let s be a fixed integer and let $U_{1}, \ldots, U_{k}$ be subsets of typical vertices of sizes $\left|U_{1}\right|=2 s$ and $\left|U_{2}\right|=\ldots=\left|U_{k}\right|=s$, which belong to $k$ different parts of the partition of $G$. Without loss of generality, suppose that $U_{i} \subset V_{i}$ and let $U=\cup_{i=1}^{k} U_{i}$ and $W=\cup_{j>k} V_{j}$. Then $G$ contains a complete bipartite graph with parts $U^{\prime} \subset U$ and $W^{\prime} \subset W$ such that $\left|U^{\prime}\right| \geq\left(k+\frac{3(r-k)-2}{3(r-k)}\right) s$ and $\left|W^{\prime}\right|=\Omega(n)$.

Proof. Since every typical vertex $x \in V_{i}$ has $d_{V_{i}}(x) \leq \epsilon n / 2$, we obtain that the number of its neighbors in $W$ is at least

$$
\begin{aligned}
d_{W}(x) & \geq d(v)-d_{V_{i}}(x)-\sum_{j \leq k, j \neq i}\left|V_{j}\right| \\
& \geq d(v)-\epsilon n / 2+\left|V_{i}\right|-\sum_{j \leq k}\left|V_{j}\right| \\
& \geq\left(\frac{3 r-4}{3 r-1}+\epsilon\right) n-\epsilon n / 2+\left|V_{i}\right|-(n-|W|) \\
& \geq|W|+\left|V_{i}\right|-\frac{3}{3 r-1} n .
\end{aligned}
$$

Note that $|W|+\sum_{i=1}^{k}\left|V_{i}\right|=n$ and also by (1) we have $|W|=\sum_{j>k}\left|V_{j}\right| \leq(r-k) \frac{3}{3 r-1} n$ and $\left|V_{1}\right| \geq\left(\frac{2}{3 r-1}+\epsilon / 2\right) n$. All these facts together give the following estimate on the number of edges between $U$ and $W$ 


$$
\begin{aligned}
e(U, W) & =\sum_{x \in U} d_{W}(x)=\sum_{i=1}^{k} \sum_{x \in U_{i}} d_{W}(x) \geq \sum_{i=1}^{k}\left(|W|+\left|V_{i}\right|-\frac{3}{3 r-1} n\right)\left|U_{i}\right| \\
& =\left((k+1)|W|+\left|V_{1}\right|+\sum_{i=1}^{k}\left|V_{i}\right|-(k+1) \frac{3}{3 r-1} n\right) s \\
& \geq\left(k|W|+\left(\frac{2}{3 r-1}+\epsilon / 2\right) n+\left(|W|+\sum_{i=1}^{k}\left|V_{i}\right|\right)-\frac{3 k+3}{3 r-1} n\right) s \\
& =\left(k|W|+\epsilon n / 2+\frac{3(r-k)-2}{3 r-1} n\right) s \\
& \geq\left(k+\frac{3(r-k)-2}{3(r-k)}\right)|W| s+\Omega(n) .
\end{aligned}
$$

Since $U$ has constant size and $d_{U}(y) \leq|U|$ for all $y \in W$, we conclude that there are at least

$$
\frac{e(U, W)-\left(k+\frac{3(r-k)-2}{3(r-k)}\right) s \cdot|W|}{|U|}=\Omega(n)
$$

vertices in $W$ whose degree in $U$ is larger than $\left(k+\frac{3(r-k)-2}{3(r-k)}\right) s$. To complete the proof, note that the number of subsets of $U$ is also bounded by a constant and therefore at least $\Omega(n)$ such vertices will have the same set of neighbors $U^{\prime}$ in $U$.

Finally we need the following simple estimate.

Lemma 2.8 For all integers $r \geq 2$ we have the following inequality

$$
\frac{1}{3} \cdot \frac{4}{6} \cdots \frac{3 r-5}{3 r-3}>\frac{1}{4 r^{2 / 3}}
$$

Proof. Let $x=\prod_{j=2}^{r-1} \frac{3 j-2}{3 j}, y=\prod_{j=2}^{r-1} \frac{3 j-3}{3 j-1}$ and let $z=\prod_{j=2}^{r-1} \frac{3 j-4}{3 j-2}$. Since $\frac{3 j-2}{3 j}>\frac{3 j-3}{3 j-1}>$ $\frac{3 j-4}{3 j-2}$ and all three products have the same number of terms we have that $x>y>z$. Therefore

$$
x^{3}>z y x=\frac{2}{4} \cdot \frac{3}{5} \cdot \frac{4}{6} \cdots \frac{3 r-7}{3 r-5} \cdot \frac{3 r-6}{3 r-4} \cdot \frac{3 r-5}{3 r-3}=\frac{2 \cdot 3}{(3 r-4)(3 r-3)}>\frac{2}{3 r^{2}} .
$$

This implies the assertion of the lemma, since $\frac{1}{3} \cdot \frac{4}{6} \cdots \frac{3 r-5}{3 r-3}=x / 3>\frac{1}{3}\left(\frac{2}{3 r^{2}}\right)^{1 / 3}>\frac{1}{4 r^{2 / 3}}$.

Having finished all the necessary preparations, we are now ready to complete the proof of Theorem 1.2. Without loss of generality, suppose that $V_{1}$ spans at least $2 n^{2-1 /\left(4 r^{2 / 3} t\right)}$ edges. By Lemma 2.4, only at most $O(n)$ of these edges are incident to non-typical vertices. Therefore the set of typical vertices in $V_{1}$ spans at least $n^{2-1 /\left(4 r^{2 / 3} t\right)}$ edges. By the well known result of Kövari, Sós and Turán [9] about the Turán numbers of bipartite graphs, $V_{1}$ contains a complete bipartite graph $H_{1}$ with parts $(A, B)$ of size $|A|=|B|=s_{1}=4 r^{2 / 3} t$ 
all of whose vertices are typical. If there are at least $s_{2}=\frac{3 r-5}{3 r-3} s_{1}$ typical vertices in one of the remaining parts $V_{2}, \ldots, V_{r}$ which are adjacent to two subsets $A^{\prime} \subset A, B^{\prime} \subset B$ of size $s_{2}$ then we add them to $\left(A^{\prime}, B^{\prime}\right)$ to form a complete 3 -partite graph $H_{2}$ with parts of sizes $s_{2}$ and continue.

Suppose that at step $1 \leq k \leq r-1$ we have a complete $k+1$-partite graph $H_{k}$ with parts $\left(A, B, U_{2}, \ldots, U_{k}\right)$ of size $s_{k}$ each, all of whose vertices are typical and $A, B \subset V_{1}$. Without loss of generality we can assume that $U_{i} \subset V_{i}$ for all $2 \leq i \leq k$. Put $U_{1}=A \cup B$ and let $U=\cup_{i=1}^{k} U_{k}$ and $W=\cup_{j>k} V_{j}$. Then, by Lemma 2.7, $G$ contains a complete bipartite subgraph with parts $\left(U^{\prime}, W^{\prime}\right)$ such that $U^{\prime} \subset U,\left|U^{\prime}\right| \geq\left(k+\frac{3(r-k)-2}{3(r-k)}\right) s_{k}$ and $W^{\prime} \subset W,\left|W^{\prime}\right| \geq \Omega(n)$. Note that, since all parts of $H_{k}$ have size $s_{k}$, we have that all intersections $U^{\prime} \cap A, U^{\prime} \cap B$ or $U^{\prime} \cap U_{i}, 2 \leq i \leq k$ have size at least $\left|U^{\prime}\right|-k s_{k} \geq \frac{3(r-k)-2}{3(r-k)} s_{k}=$ $s_{k+1}$. Also, since $\left|W^{\prime}\right| \geq \Omega(n)$ and there are at most $O(1)$ non-typical vertices, there exists an index $j>k$ such that $W^{\prime} \cap V_{j}$ contains at least $s_{k+1}$ typical vertices. Let $U_{k+1}^{\prime}$ be some set of $s_{k+1}$ typical vertices from $W^{\prime} \cap V_{j}$. Choose subsets $A^{\prime} \subset U^{\prime} \cap A, B^{\prime} \subset U^{\prime} \cap B$ and $U_{i}^{\prime} \subset U^{\prime} \cap U_{i}, i \leq k$ all of size $s_{k+1}$. Then $\left(A, B, U_{2}, \ldots, U_{k+1}\right)$ form a complete $k+1$-partite graph $H_{k+1}$ with parts of size $s_{k+1}$ all of whose vertices are typical.

Continuing the above process $r-1$ steps we obtain a complete $(r+1)$-partite graph with parts of sizes

$$
s_{r}=\frac{1}{3} s_{r-1}=\frac{1}{3} \cdot \frac{4}{6} s_{r-2}=\ldots=\frac{1}{3} \cdot \frac{4}{6} \cdots \frac{3 r-5}{3 r-3} s_{1}>\frac{s_{1}}{4 r^{2 / 3}}=t .
$$

This contradicts our assumption that $G$ is $K_{r+1}(t)$-free and shows that every $V_{i}$ spans at most $O\left(n^{2-1 /\left(4 r^{2 / 3} t\right)}\right)$ edges. Therefore the number of edges we need to delete to make $G$ $r$-partite is bounded by $\sum_{i} e\left(V_{i}\right) \leq O\left(n^{2-1 /\left(4 r^{2 / 3} t\right)}\right)$. This completes the proof of Theorem 1.2.

Acknowledgment. We would like to thank Asaf Shapira for helpful discussions.

\section{References}

[1] N. Alon, A. Shapira and B. Sudakov, Additive approximation for Edge-deletion problems, Proc. 46 ${ }^{\text {th }}$ IEEE FOCS, IEEE (2005), 419-428.

[2] B. Andrásfai, P. Erdős and V. Sós, On the connection between chromatic number, maximal clique and minimal degree of a graph, Discrete Math. 8 (1974), 205-218.

[3] P. Erdős, On extremal problems of graphs and generalized graphs, Israel J. Math. 2 (1964), 183-190.

[4] P. Erdős and M. Simonovits, A limit theorem in graph theory, Studia Sci. Math. Hungar 1 (1966), 51-57.

[5] P. Erdős and M. Simonovits, On a valence problem in extremal graph theory, Discrete Math. 5 (1973), 323-334. 
[6] P. Erdős and A. Stone, On the structure of linear graphs, Bull. Amer. Math. Soc. 52 (1946), 1087-1091.

[7] Z. Füredi, Turán type problems, in: Surveys in combinatorics, London Math. Soc. Lecture Note Ser. 166, Cambridge Univ. Press, Cambridge, 1991, 253-300

[8] J. Komlós and M. Simonovits, Szemerédi's Regularity Lemma and its applications in graph theory, in: Combinatorics, Paul Erdős is eighty, Vol. 2, János Bolyai Math. Soc., Budapest, 1996, 295-352.

[9] T. Kövari, V.T. Sós and P. Turán, On a problem of K. Zarankiewicz,Colloquium Math. 3 (1954), 50-57.

[10] E. Szemerédi, Regular partitions of graphs, in: Proc. Colloque Inter. CNRS 260, CNRS, Paris, 1978, 399-401.

[11] P. Turán, Eine Extremalaufgabe aus der Graphentheorie, Mat. Fiz Lapok 48 (1941), 436-452. 